\title{
Anisotropic responses to motion toward and away from the eye
}

\author{
JOHN A. PERRONE \\ NASA Ames Research Center, Moffett Field, California
}

\begin{abstract}
When a rigid object moves toward the eye, it is usually perceived as being rigid. However, in the case of motion away from the eye, the motion and structure of the object are perceived nonveridically, with the percept tending to reflect the nonrigid transformations that are present in the retinal image. This difference in response to motion to and from the observer was quantified in an experiment using wire-frame computer-generated boxes which moved toward and away from the eye. Two theoretical systems are developed by which uniform three-dimensional velocity can be recovered from an expansion pattern of nonuniform velocity vectors. It is proposed that the human visual system uses two similar systems for processing motion in depth. The mechanism used for motion away from the eye produces perceptual errors because it is not suited to objects with a depth component.
\end{abstract}

Self-motion through a rigid environment at constant velocity generates an expansion pattern of velocity vectors on the retina. The retinal velocity of points close to the eye is greater than that for more distant points. Despite this nonuniform distribution of velocity vectors, the resulting percept tends to be veridical, in the sense that the velocity of points in three-dimensional (3-d) space appears equal and constant. At least, this is the case for motion toward the eye.

If we view the receding scene from the rear of a moving vehicle, we notice that points close to the eye appear to be moving away at a greater velocity than do points in the distance. Objects seem to suddenly decelerate as they appear at the edges of the visual field and move away from the eye. This same effect can be seen when the observer is stationary and objects, such as cars, pass close by, moving in the same direction as the line of gaze. For motion away from the eye, the percept tends to reflect the nonuniform, nonrigid projection present in the twodimensional (2-d) retinal image.

It is not too surprising to discover that the human visual system has an anisotropic response to motion in depth, since anisotropies for 2-d motion responses have often been demonstrated (Ball \& Sekuler, 1980; Georgeson \& Harris, 1978), and 2-d retinal motion forms the basis of 3-d velocity extraction.

Another reason to suspect, a priori, the existence of anisotropic responses to motion in depth is that humans evolved with a forward-facing visual system, not a backward-facing one. It is only recently that man has been able to move at high velocities through his environment

This research was carried out while the author was on a National Research Council Associateship. Thanks are extended to Denis Pelli for his helpful comments on an earlier version of this paper. The author's mailing address is: NASA Ames Research Center, MS 239-3, Moffett Field, CA 94035. while facing backward, or to have large rigid objects move rapidly away from him.

The experiment reported in this paper was designed to verify and quantify the phenomenological differences observed in response to motion toward the eye versus motion away from the eye.

See Figure 1. If an observer views monocularly a long rigid box moving along the axis of gaze, the retinal projection is such that the 2-d velocities of points on the front and back faces are quite different. The actual size of the difference depends on the length of the box, as dictated by the laws of perspective.

By manipulating the relative 3-d velocities of the near and far faces of a simulated box moving in depth, it is possible to determine the point at which a 2 -d display of the box appears rigid. This is the point at which no relative movement is detectable between any parts of the box as it moves toward or away from the eye. If the velocity of the near face of the box $\left(V 3 D_{1}\right)$ is not equal to that of the far face $\left(V 3 D_{2}\right)$, the 2-d display would represent a nonrigid box that is expanding or contracting as it moves toward or away from the observer. The observer must determine if $V 3 D_{1}=V 3 D_{2}$ in Figure 1 , solely from the information in the $2-d$ projection. To carry out such a task, the observer needs to extract information concerning the relative velocities of points in 3-d space. The question is, can this be done equally well for boxes moving toward the eye and for those moving away from the eye?

\section{METHOD}

The stimuli were 2-d perspective representations of boxes moving along the line of sight. These were wire-frame computergenerated figures produced on a Hewlett-Packard 1345A vector graphics display using a refresh rate of $60 \mathrm{~Hz}$. This display has a flat screen, $9.5 \mathrm{~cm}$ high and $12.5 \mathrm{~cm}$ wide. It was viewed monocularly from a distance of $57.3 \mathrm{~cm}$ through an $18^{\circ}$ diam circular aperture in an occluding screen $36 \mathrm{~cm}$ from the face of the display. The 


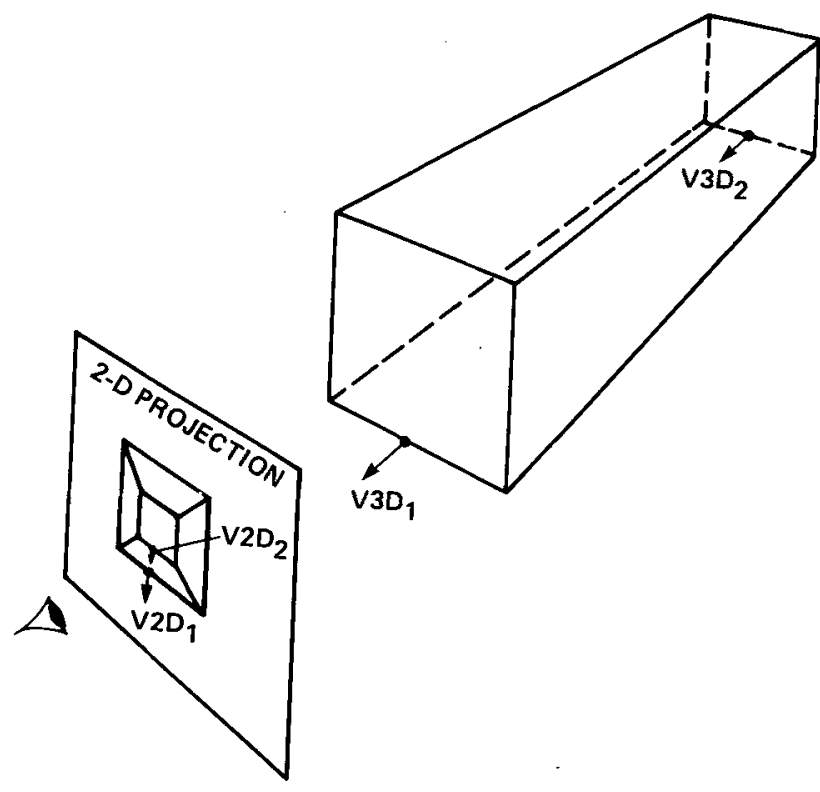

Figure 1. Subjects viewed monocularly a two-dimensional (2-d) computer-generated display depicting a box moving toward or away from the eye, and had to decide if the box was rigid or not (i.e., does $V 3 D_{1}=V 3 D_{2}$ ?) on the basis of information in the 2-d display.

area behind the occluding screen was darkened such that the stimuli appeared as luminous wire-frame figures against a black background. From the correct eye-point, the stimuli represented boxes measuring $16 \mathrm{~cm}$ high $\times 16 \mathrm{~cm}$ wide and three different depths: 100,200 , and $300 \mathrm{~cm}$.

For the motion-toward condition, the near face began at a simulated distance of $250 \mathrm{~cm}$ from the eye and moved at a constant velocity of $50 \mathrm{~cm} / \mathrm{sec}$ for $3 \mathrm{sec}$ to finish up at a distance of $100 \mathrm{~cm}$ from the eye. The velocity of the far face was always constant during movement of the box, but its value could be changed from trial to trial. If it was less than $50 \mathrm{~cm} / \mathrm{sec}$, then the stimulus for that trial would represent a nonrigid box which stretched in length as it approached the eye. For the motion-away condition, the near face of the box started at $100 \mathrm{~cm}$ from the eye. This time, the far face moved for $3 \mathrm{sec}$ at $50 \mathrm{~cm} / \mathrm{sec}$ and the velocity of the near face was variable from trial to trial. For the two conditions, the front, or leading, face was always the standard $(50 \mathrm{~cm} / \mathrm{sec}$ velocity) and the trailing face was the variable.

Five volunteer subjects were used, all naive as to the aims of the experiment. They all had normal or corrected-to-normal visual acuity. The subject was seated at a headrest with his/her nonpreferred eye occluded by an eye patch. The subject initiated a trial by pressing a key on a keyboard, and the stimuli appeared and began moving instantly. There was no fixation point in the display. The stimuli disappeared immediately following the $3 \mathrm{sec}$ of movement.

For the motion-toward condition, the subject responded "Yes" if the box appeared to expand as it approached (far face moving more slowly than near face) or "No" if it appeared to be rigid or contracting (velocity of far face same as or greater than that of near face). For the motion-away condition, the subject responded "Yes" if the box appeared to contract as it moved away (near face moving faster than far face) or "No" if the box appeared to be rigid or expanding (velocity of near face same as or less than that of far face).

For each of the two main conditions (motion-toward, motionaway), three levels of box length were used $(100,200,300 \mathrm{~cm})$, giving six experimental conditions, which were presented in random order. A double interleaved staircase procedure was used for each condition, and a session was completed after 8 reversals of each staircase. The first 2 reversals of each staircase were discarded, and the mean for each subject was based on the 12 remaining reversals. The means reflect the velocity of the variable relative to the standard $(50 \mathrm{~cm} / \mathrm{sec})$ for which the box was judged on $50 \%$ of the trials to be no longer expanding (motion-toward condition) or contracting (motion-away condition). Presumably, at these points the stimuli were indistinguishable from rigid boxes.

\section{RESULTS}

\section{Motion Toward the Eye}

The mean setting for the velocity of the variable (far face) for each of the 5 subjects was converted into a percentage of the velocity of the standard (near face). The mean values from the 5 subjects were pooled and plotted as a function of the box length in Figure 2a. The $100 \%$ line represents the point at which the 3 -d velocities of the near and far faces were equal. The data suggest that the boxes were perceived as rigid when the 3-d velocity of the variable was very close to the velocity of the standard. Thus, for motion toward the eye, the subjects were able to correctly extract the 3-d depth information from the 2-d stimulus.

If the subjects based their responses directly upon the velocities in the 2-d image, the data points in Figure $2 \mathrm{a}$ would be $150 \%, 200 \%$, and $260 \%$ for the $100-, 200-$,
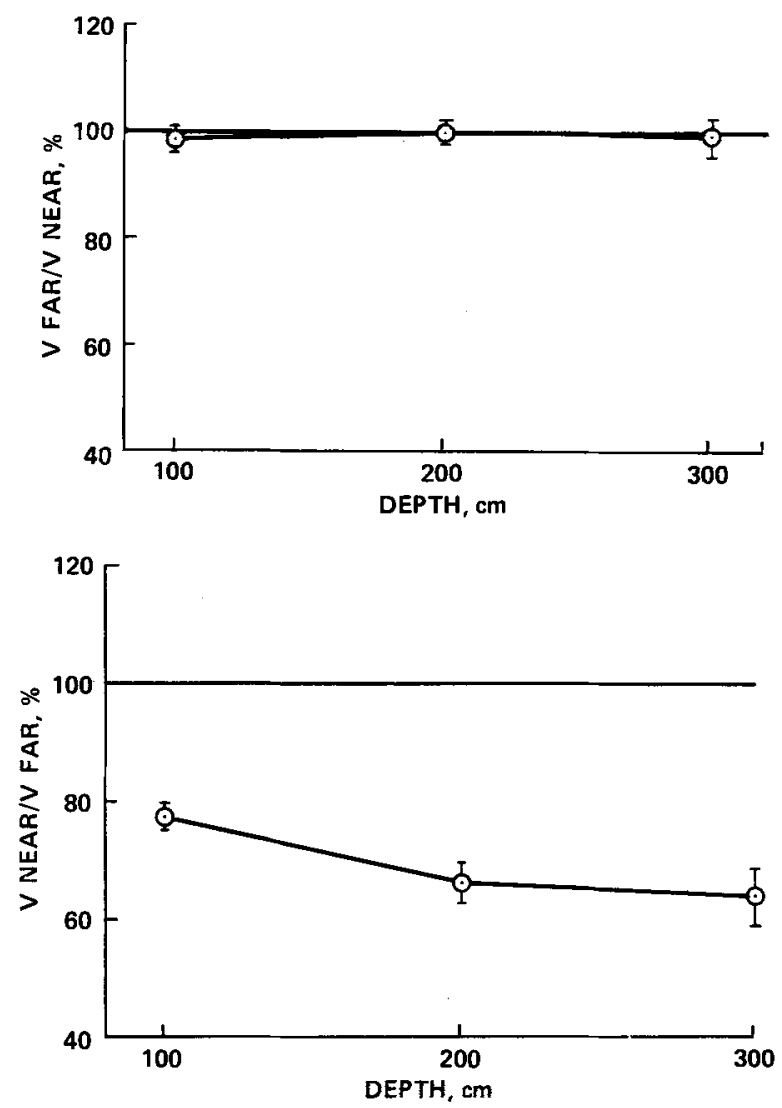

Figure 2. Mean values for velocity of variable face relative to standard face, when the boxes were judged to be rigid. (a) Motion toward the eye. (b) Motion away from the eye. 
and $300-\mathrm{cm}$ boxes, respectively. These are the approximate values that would be required to match the $2-d$ velocities of points on the near and far faces of the boxes. They are only approximate because the 2 -d velocities are not constant, and it is not possible to match the velocities over the whole $3 \mathrm{sec}$ of movement. The actual data points lie well below these values, and so we can conclude that the subjects were not simply attempting to match the 2-d velocities in the stimuli.

\section{Motion Away From the Eye}

The data for the motion-away condition are shown in Figure $2 \mathrm{~b}$. The ordinate is again the mean velocity of the variable expressed as a percentage of the standard velocity. However, in this case, the variable was the near face and the standard was the far face. For this condition, it is apparent that in order for the box to appear rigid, the velocity of the near face had to be slowed somewhat relative to the far face. These data support the observation that points close to the eye appear to move faster than points far from the eye, when the motion is away from the observer and the points are, in fact, moving at the same velocity.

A test for linear trend was significant $[F=26.2$; F.99 $(1,8)=11.3$, which indicates that the errors increased as the box length increased. At first glance, this result suggests that the subjects were attempting to match the 2-d velocities of points in the stimulus display, because the longer the box, the greater the difference between the 2-d velocities of points on the near and far faces. However, the approximate values that would be obtained if subjects attempted to match the 2-d velocities are $24 \%$, $10 \%$, and $6 \%$ for the $100-, 200-$, and $300-\mathrm{cm}$ conditions, respectively. These values are much smaller than the obtained means, and so it is unlikely that the data represent a complete breakdown in the extraction of 3-d depth information. The departure of the data from the $100 \%$ line, however, is evidence of an anisotropic response to motion in depth, because the data for the case of motion toward the eye did not display such a deviation.

\section{THEORETICAL 3-d MOTION ANALYSIS}

Before we can attempt to determine why the visual system incorrectly processes motion in depth away from the eye, we need to understand how it correctly analyzes motion toward the eye. A lot of interest has recently been shown in the structure-from-motion problem (e.g., Longuet-Higgins, 1981; Prazdny, 1980; Tsai \& Huang, 1984), but the simpler issue of how uniform 3-d velocity can be extracted from a nonuniform 2-d expansion pattern has not been specifically addressed. The problem is analogous to the case of size constancy in which identical objects at different distances produce different-sized retinal images, yet they tend to be perceived as being equal.

Two methods will be presented for solving this problem. The first is more general but also more complex. The sec- ond uses assumptions about the environment to simplify the computation.

\section{System 1}

Consider the case depicted in Figure 3a with the eye located a distance, $h$, above a plane and the gaze-line directed at an arbitrary angle $\phi$ degrees below the horizon for that plane. The direction $\mathrm{OH}$ in Figure 3a is parallel to the plane, and it represents the direction of the "horizon." The 2-d information reaching the eye will be analyzed on a theoretical projection plane an arbitrary distance, $f$, from the eye. The value of $f$ is a known constant, and it corresponds to the distance from the principal plane of the cornea of the eye to the retina, if the retina is used as the projection plane.

At a particular instant of time, a point moving at constant velocity will be at a horizontal distance, $Z$, out from the eye. The point $P$ projects onto the projection plane with a 2-d velocity equal to $V_{2 D}$. All the points lying on the same plane as $P$ and moving at the same velocity as $P$ will project as a distribution of velocity vectors typically called an "expansion pattern." This is represented in Figure $3 b$.

Points on the plane that are at a very large horizontal distance from the eye will project as having very small velocity vectors. In fact, the length of the vectors reaches a limit (zero) at the point at which the line $\mathrm{OH}$ in Figure 3a intersects with the projection plane. This limit is referred to as the focus of expansion (FOE), and it is shown as $a$ dot at the top of Figure $3 b$.

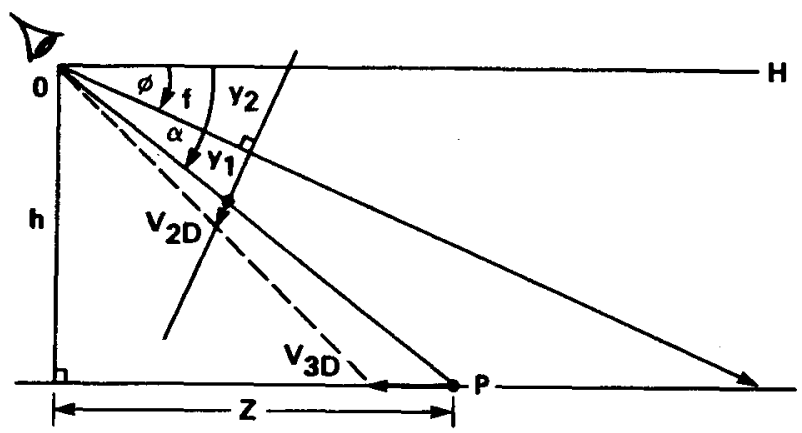

(a)

(b)

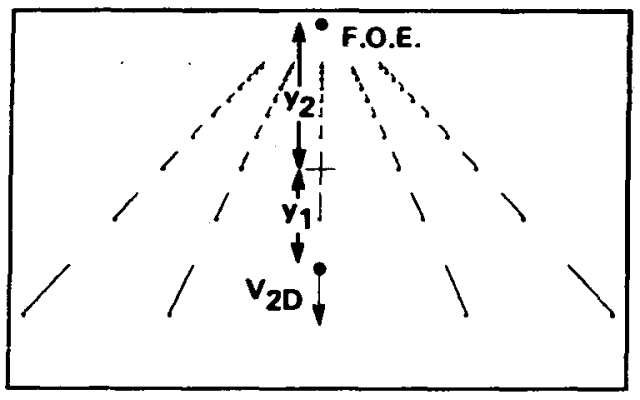

Figure 3. Analysis of the 2-d information reaching the eye. Equation 1 enables the original uniform 3-d velocity of the point $P$ to be recovered from the $2-d$ expansion pattern. 
The point at which the line of sight intersects with the projection plane will be referred to as the center of the projection plane, and it corresponds to the position of the fovea. It is marked as a " + "' in Figure 3b.

Analysis of the 2-d information reaching the eye (see Appendix) establishes the following relationship:

$$
\mathrm{V}_{3 \mathrm{D}}=\frac{-\mathrm{hf}}{\left(\sin ^{2} \alpha\right)\left(\mathrm{f}^{2}+\mathrm{y}_{1}^{2}\right)} \mathrm{V}_{2 \mathrm{D}}
$$

where $\alpha=\tan ^{-1}\left(y_{1} / f\right)+\tan ^{-1}\left(y_{2} / f\right) ; y_{1}$ is the distance measured within the projection plane, from the current position of the projected point to the center of the projection plane; and $y_{2}$ is the distance from the center of the projection plane to the focus of expansion.

The expression on the right side of Equation 1 remains constant whenever $V_{3 D}$ is constant. Therefore, despite the fact that the value of $V_{2 D}$ changes as $P$ moves closer or further away from the eye, an invariant property of the $2-d$ stimulus can be extracted which indicates that $P$ is moving at constant velocity in 3-d space. Similarly, points on the plane at different distances from the eye will generate the same value from Equation 1, even though their $2-d$ velocities are different.

For simplicity, a point $P$ lying in the vertical plane passing through the eye has been used for the analysis. However, Equation 1 is general, and it can be applied to any point in the expansion pattern as long as the appropriate components of the velocity vectors are used for the values of $y_{1}$ and $y_{2}$.

Equation 1 shows that in theory, it is possible to recover uniform 3-d velocity from a 2-d expansion pattern. If we know the values of $f$ and $h$, it is possible to recover the original absolute value of the 3 -d velocity.

This scheme requires some means of detecting the 2-d velocities and the ability to determine the position of the focus of expansion. Given two noncollinear points, the focus of expansion can be determined from the intersection of their extended velocity vectors.

With these two properties, the human visual system could extract uniform 3-d velocities from a nonuniform 2-d retinal projection using a scheme similar to this theoretical one.

\section{System 2}

There is another system whereby $3-d$ constant velocity can be extracted from a $2-\mathrm{d}$ retinal projection, but this works only for points lying in a plane orthogonal to the direction of motion (see Figures $4 \mathrm{a}$ and $4 \mathrm{~b}$ ). For simplicity, the case in which the gaze-line is parallel to the direction of motion will be used, but the same result can be obtained for the more general situation in which the direction of motion and gaze-line are nonparallel.

Consider any two points moving at the same uniform velocity and lying in a plane orthogonal to the direction of motion. At a particular instant of time, $t$, they are at distance $Z$ from the eye and project onto the projection plane at $p_{1}$ and $p_{2}$. Let the actual spacing between the

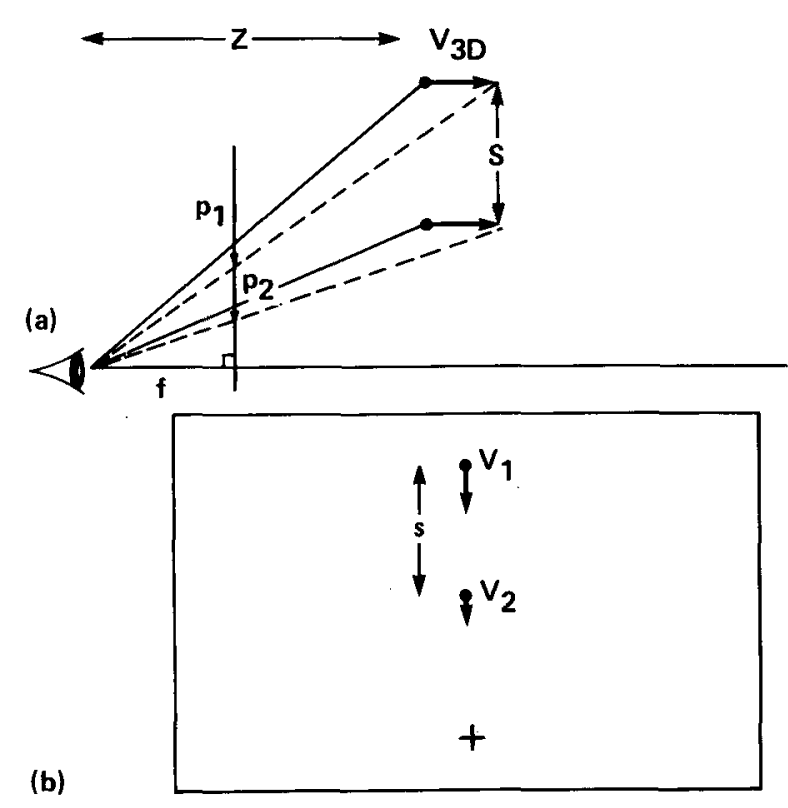

Figure 4. The case in which two points lie in a plane orthogonal to the direction of motion. The 3-d velocity can be recovered from the 2-d projection using Equation 3. This system is not dependent upon knowing the position of the focus of expansion.

points be $S$, and the spacing between the projected points be $s$. The points always remain at the same distance apart; that is, $\mathrm{S}$ is constant. The arbitrary distance from the eye to the projection plane is $f$.

Now the horizontal distance of the points from the eye is $\mathrm{Z}=\mathrm{fS} / \mathrm{s}$, so

$$
\frac{\mathrm{dZ}}{\mathrm{dt}}=-\frac{\mathrm{fS}}{\mathrm{s}^{2}} \frac{\mathrm{ds}}{\mathrm{dt}} .
$$

$\mathrm{dZ} / \mathrm{dt}$ is the 3-d velocity, $\mathrm{V}_{3 \mathrm{D}}$, of the points as they move toward or away from the eye. $\mathrm{ds} / \mathrm{dt}$ is the rate at which the spacing between $p_{1}$ and $p_{2}$ changes over time, and it is given by the difference in the 2-d projected velocities of the two points $\left(V_{1}-V_{2}\right)$. With these substitutions, Equation 2 can be rewritten as:

$$
\mathrm{V}_{3 \mathrm{D}}=-\mathrm{fS} \frac{\mathrm{V}_{1}-\mathrm{V}_{2}}{\mathrm{~s}^{2}}
$$

Thus, we can recover the original 3-d velocity of the two points from the 2-d projection by taking the difference of their respective 2-d velocities, dividing by the square of the distance between them, and multiplying by a constant ( $-\mathrm{fS})$. This quantity remains invariant over time as the points move toward or away from the eye at constant velocity.

We can think of this technique as a local method of analysis, because it does not require knowledge of the position of the focus of expansion. It is a simpler system, but it works only for points at the same depth. This method produces errors when attempts are made to apply it to the case in which points lie at different distances from the eye. 
Modeling the Anisotropic Response to 3-d Motion

Given these two systems for extracting 3-d motion from a 2-d expansion pattern, it is possible to model the anisotropic response to motion in depth by proposing that System 1 is used for motion toward the eye and System 2 is used for motion away from the eye. This is not to say that the human visual system uses these exact methods for 3-d motion analysis, but rather that the same basic variables are used.

The characteristic feature of the anisotropic response to 3-d motion is that it occurs whenever objects having a depth component move away from the eye. The experimental stimuli consisted of boxes with front and back faces at different distances from the eye. This is not problematical to a technique such as System 1, and so, for motion toward the eye, we would expect the 3-d motion to be correctly registered. For motion away from the eye, however, we would expect errors to occur, because System 2 cannot accommodate points with different depth values.

For the experimental stimuli, $\left(\mathrm{V}_{1}-\mathrm{V}_{2}\right) / \mathrm{s}^{2}$ remains constant for any two points on the near face of the box and for any two points on the far face, but for a point on the near face and a point on the far face it changes over time. The stimuli would not appear rigid to a visual system using Equation 3 to extract 3-d velocity. It is not immediately obvious from the theory as to how the changing value of $\left(V_{1}-V_{2}\right) / s^{2}$ manifests itself perceptually. One interpretation is that the value of $s$ (the spacing between points on the near and far faces) is decreasing too rapidly relative to other dimensions in the figure.

\section{DEPTH $=100 \mathrm{~cm}$}

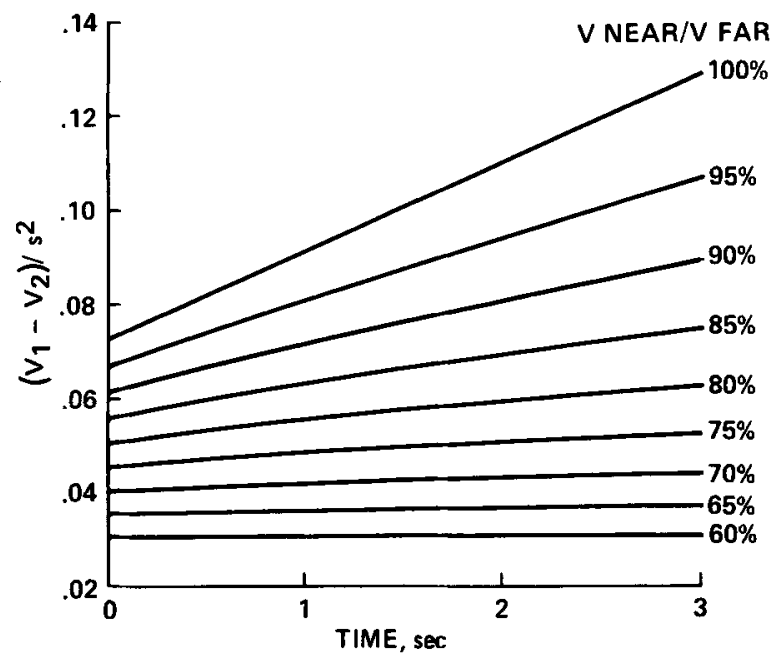

Figure 5. Plot showing how the rigidity index $\left(V_{1}-V_{2} / s^{2}\right)$ changes over the $3 \mathrm{sec}$ of movement of the $100-\mathrm{cm}$ box away from the eye. When the velocity of the near face is reduced to approximately $60 \%$ of that of the far face, the RI remains fairly constant over time, and so the box should appear rigid.

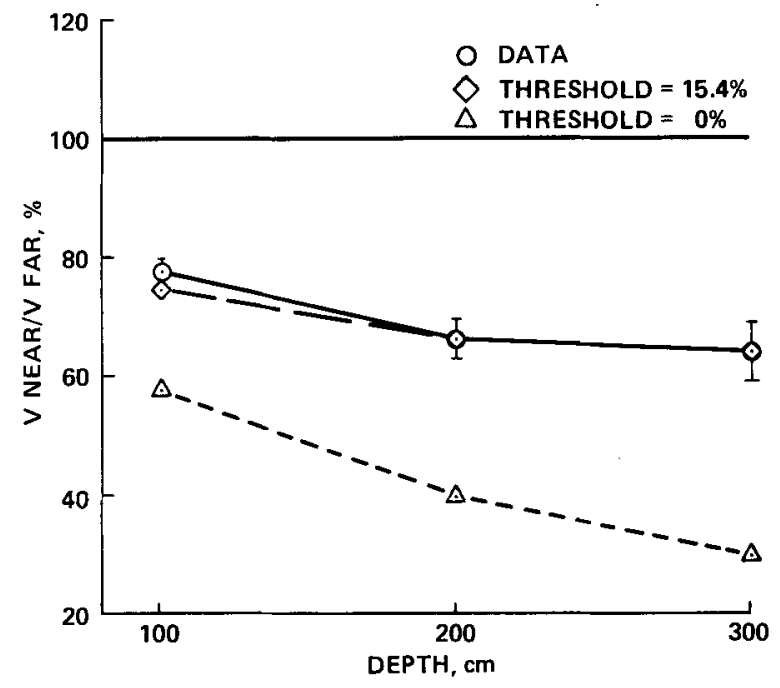

Figure 6. Predictions and data. The triangles represent the predictions of the model if perfect performance is assumed. If the value of the threshold is fixed to that of the $200-\mathrm{cm}$ box $(15.4 \%)$, the predictions of the model for the 100 - and $300-\mathrm{cm}$ boxes are shown as diamonds.

For convenience, the expression $\left(\mathrm{V}_{1}-\mathrm{V}_{2}\right) / \mathrm{s}^{2}$ will be referred to as the "rigidity index" (RI). Figure 5 shows just how the value of the rigidity index changes during the $3 \mathrm{sec}$ of movement of the box away from the eye. This is for the case of the $100-\mathrm{cm}$ box. When both the near and far faces were moving at the same 3-d velocity (shown by the $100 \%$ line in Figure 5), we see that the rigidity index increases over time and so the box should appear nonrigid. However, as the velocity of the near face is slowed relative to the far face, we note that the RI gets closer to being constant. If the human visual system were using this type of analysis for extracting 3-d velocity, we would predict that, when the velocity of the near face got down to about $60 \%$ of the far face, the box would start to look rigid.

The $200-$ and $300-\mathrm{cm}$ boxes have slightly different patterns of change, and the percentages are $40 \%$ and $30 \%$, respectively, in order for the slope of the functions to reach zero. These are the predictions for the experimental data under the motion-away condition and are shown as triangles, along with the data in Figure 6. However, these predictions are for perfect performance, with the assumption that subjects are able to detect the slightest change in the value of the RI as the box moves away from the eye.

The mean data value for the $200-\mathrm{cm}$ box was $66.2 \%$; that is, the near face was moving at only $66.2 \%$ of the far face when the box was judged to be rigid. By using a point on the near face and a point on the far face, we can calculate, for this case, the value of the RI at the start of the movement and for the end of the movement $3 \mathrm{sec}$ later. (These values would be represented graphically by the endpoints of the $66.2 \%$ line in the equivalent $200-\mathrm{cm}$ figure to Figure 5.) We find that the rigidity index in- 
creased by $15.4 \%$ during the $3 \mathrm{sec}$ of movement away from the eye. If we adopt $15.4 \%$ as a "detection threshold" and apply this threshold to the 100- and 300$\mathrm{cm}$ conditions, we can calculate what the velocity of the near face must be relative to the far face in order to generate a $15.4 \%$ change in the RI. These are the predictions for the 100 - and $300-\mathrm{cm}$ data when it is assumed that the smallest change that can be detected in the RI is $15.4 \%$ over the $3 \mathrm{sec}$ of movement. These predictions are shown as diamonds in Figure 6.

The close correspondence of the predictions with the data demonstrate that it is possible to model the 3-d motion effect by assuming that 3-d velocity information is extracted using a mechanism such as System 2 , when motion is away from the eye.

\section{DISCUSSION}

Some support has been gained for the proposal that two different mechanisms are used by the human visual system to extract 3-d velocity from a 2-d expansion pattern. The fact that subjects made essentially no errors in the case of motion toward the eye suggests that some invariant property of the stimulus pattern was being extracted. The invariant outlined in System 1 is one possibility.

Because subjects did not correctly perceive the boxes to be rigid when they moved away from the eye, we can conclude that the invariant properties of the expansion pattern were not correctly extracted. It may be that the visual system is trying to carry out an analysis such as System 1 , but that errors occur because some variables are incorrectly registered. More likely, the visual system uses an entirely different mechanism for the case of motion away from the eye. The close fit between the predictions and the experimental data supports the latter view and points to the possibility that a mechanism such as System 2 is used in the case of motion away from the eye.

If we are to conclude that two different mechanisms are used by the visual system to analyze motion in depth, then we need to consider how the inputs to these two systems differ. If the eye always foveated in the direction of motion, then motion toward the eye would be characterized by $2-d$ velocity vectors that expanded radially out from the fovea, and motion away from the eye would generate velocity vectors in toward the fovea. It would then be a simple matter to postulate that motion detectors tuned to centrifugal motion feed into a System 1 type analyzer and that those tuned to centripetal motion are part of a System 2 type analyzer. Unfortunately, the direction of gaze does not always coincide with the direction of movement, and it is possible to have the situation in which points in the expansion pattern move in toward the fovea, even though the motion is toward the eye.

There is no classification scheme, based on direction of movement, that can be applied to a single moving point to differentiate motion toward the eye from motion away from the eye. It is possible, however, to develop such a scheme based on the $2-d$ motion of two points. If the $2-d$ velocity vectors of two points are diverging, then the points are most likely to be moving toward the observer in 3-d space. The point of convergence of these vectors, when extrapolated backward, would indicate the position of the focus of expansion. The analysis of 3-d motion in this case would be handled by a System 1 type analyzer. If the 2-d velocity vectors are converging, then the points are moving away from the observer in 3-d space and the motion would be analyzed by a System 2 type mechanism, which does not compute the position of the focus of expansion.

The requirement is for two channels selectively sensitive to increasing and to decreasing size. Regan and Beverley $(1978,1979)$ have proposed and provided psychophysical evidence for the existence of two such channels. More recently, Toyama, Komatsu, Kasai, Fujii, and Umetani (1985) measured the responsiveness of Clare-Bishop neurons in the cat to 3-d motion and found cells that were selectively responsive to motion toward and away from the eye. Their main study concerned motion disparity in two eyes, but similar specialized cells were found in the case of monocular presentation. They also found some cells that were selectively responsive to an increase in stimulus size and some responsive only to a decrease in size.

The analysis given in Systems 1 and 2 concentrated on invariants involving the instantaneous $2-d$ retinal velocities of points. It is also possible to develop similar invariants with respect to the changing 2 -d velocities-namely, the acceleration or deceleration of points in the image (see Andersen \& Braunstein, 1985). It has been found that when the acceleration of points in a 2-d display is too high, observers experience a reduced impression of motion in depth (Andersen \& Braunstein, 1985; von Hofsten, 1974). Von Hofsten (1974) suggested that the most critical phase in correctly perceiving motion in depth was the initial formation of the depth percept. He found that once the percept of motion in depth was formed, much greater variation in the 2-d acceleration of points could be tolerated while maintaining the impression of motion in depth. Von Hofsten postulated that there might be a short delay in the formation of the percept.

For motion toward the eye, the greatest acceleration of points in the image occurs at the end of the object motion when it is close to the eye. For motion away from the eye, the greatest deceleration of points occurs at the very start of the motion, when the object is just moving away from the eye. There is a difference in the amount of "lead time" that occurs in each of the two cases before the visual system is required to anlayze high rates of change in the 2-d velocity of points in the image. Such a difference may provide another explanation for the anisotropic response to motion in depth, and such temporal factors need to be more fully investigated.

A related issue in the spatial domain was raised by Andersen and Braunstein (1985) to explain reduced impressions of motion in depth. This is the problem of motion correspondence. For motion toward the eye, the frame- 
to-frame position changes of individual points in the display are small at the start of the motion, whereas for motion away from the eye the initial position changes are relatively large. The latter situation may cause problems to a system that tries to establish correspondence between points in successive images, and it could also provide the basis for anisotropic responses to motion in depth.

\section{CONCLUSIONS}

The experimental data suggest that motion away from the eye is not analyzed in the same way as motion toward the eye. We perceive the motion of certain rigid objects moving away from the eye nonveridically, with the percept tending to reflect the nonrigid transformations that are present in the retinal image. It was postulated that 3$\mathrm{d}$ motion information was incorrectly extracted from the $2-\mathrm{d}$ projections, because the mechanism used by the human visual system for motion away from the eye is not suited to objects with a depth component.

The main proposal concerns the existence of two separate systems for processing motion toward the eye and motion away from the eye. The exact nature of these systems cannot be finalized on the basis of one experiment, but the theoretical model that has been presented provides a framework for future research and indicates some of the important variables present in the 2-d retinal image.

Backward locomotion is an uncommon activity in humans, and so it would not make sense in terms of the development of the visual system to include a sophisticated mechanism for dealing with 3-d motion of points at different distances from the eye. A primitive system which can detect the presence of motion toward or away from the eye would suffice. The mechanism of System 2 would be suitable, and it can deal with "flat" objects such as faces and hands which occasionally move away from the eye. Of course, such a system will be prone to errors when confronted with objects and surfaces that extend out from the eye in the third dimension.

\section{REFERENCES}

Andersen, G. J., \& Braunstein, M. L. (1985). Induced self-motion in central vision. Journal of Experimental Psychology: Human Perception \& Performance, 11, 122-132.

Ball, K., \& SEKUIER, R. (1980) Human vision favors centrifugal motion. Perception, 9, 317-325.

Georgeson, M. A., \& Harris, M. G. (1978). Apparent foveofugal drift of counterphase gratings. Perception. 7, 527-536.

LONGUET-HigGins, H. C. (1981). A computer algorithm of reconstructing a scene from two projections. Nature, 293, 133-135.

PrazdNy, K. (1980). Egomotion and relative depth map from optical flow. Biological Cybernetics, 36, 87-102.

Regan, D., \& BeverLey, K. I. (1978). Looming detectors in the human visual pathway. Vision Research, 18, 415-421.

Regan, D. \& Bevertey, K. I. (1979). Binocular and monocular stimuli for motion in depth: Changing-disparity and changing-size feed the same motion-in-depth stage. Vision Research, 19, 1331-1342.

Toyama, K., Komatsu, Y., Kasai. H., Fuji, K., \& Umetani, K.
(1985). Responsiveness of Clare-Bishop neurons to visual cues associated with motion of a visual stimulus in three-dimensional space. Vision Research, 25, 407-414.

TSAI, R. Y., \& HuANG, T. S. (1984). Uniqueness and estimation of three-dimensional motion parameters of rigid objects with curved surfaces. IEEE Transactions on Pattern Analysis \& Machine Intelligence, PAMI 6, 13-27.

von Hofsten, C. (1974). Proximal velocity change as a determinant of space perception. Perception \& Psychophysics, 15, 488-494.

\section{APPENDIX \\ Derivation of Equation 1}

In Figure $3 \mathrm{a}$, we have an eye located a distance, $h$, above a plane and the gaze-line directed at an arbitrary angle, $\phi$, below the horizon for that plane. At a particular instant of time, $t$, a point, $\mathrm{P}$, on the plane moving at constant velocity will be at a distance, $Z$, out from the base of the perpendicular from the eye to the plane.

Let $\alpha$ be the angle POH in Figure 3a. If we express the instantaneous velocity of the point $\mathrm{P}$ as the derivative, $\mathrm{dZ} / \mathrm{dt}$, and make use of the chain rule for differentiation, we can write

$$
\frac{\mathrm{dZ}}{\mathrm{dt}}=\frac{\mathrm{dZ}}{\mathrm{d} \alpha} \frac{\mathrm{d} \alpha}{\mathrm{dt}} .
$$

Now $\mathrm{Z}=\mathrm{h} \cot \alpha$, and so

$$
\frac{\mathrm{dZ}}{\mathrm{d} \alpha}=\frac{-\mathrm{h}}{\sin ^{2} \alpha} .
$$

We want to express $\alpha$ in terms of the dimensions on the projection plane, so:

$$
\alpha=\arctan \frac{y_{1}}{f}+\arctan \frac{y_{2}}{f},
$$

where $y_{1}$ is the distance measured within the projection plane, from the current position of the projected point to the center of the projection plane (see Figure $3 b$ ), and $y_{2}$ is the distance from the center of the projection plane to the point of intersection of the line $\mathrm{OH}$ with the projection plane. This point represents the position of the focus of expansion for points moving along the plane. The length $y_{2}$ is dependent upon the direction of the gaze-line (angle $\phi$ ), and it is constant as long as $\phi$ remains constant.

In order to find $\mathrm{d} \alpha / \mathrm{dt}$, we can again use the chain rule and note that:

$$
\frac{\mathrm{d} \alpha}{\mathrm{dt}}=\frac{\mathrm{d} \alpha}{\mathrm{dy}} \frac{\mathrm{dy}_{1}}{\mathrm{dt}} .
$$

Using Equation iii, we can show that:

$$
\frac{\mathrm{d} \alpha}{\mathrm{dy} \mathrm{y}_{1}}=\frac{\mathrm{f}}{\mathrm{f}^{2}+\mathrm{y}_{1}^{2}} .
$$

The expression, $\mathrm{dy}_{\mathrm{l}} / \mathrm{dt}$ in Equation iv is the rate at which the distance $y_{1}$ is changing over time. In other words, it is the instantaneous 2-d velocity of the projection of the point, given by the velocity vector in the expansion pattern. Now, substituting Equations ii, iv, and $v$ into Equation i gives:

$$
\frac{\mathrm{dZ}}{\mathrm{dt}}=\frac{-\mathrm{h}}{\sin ^{2} \alpha} \frac{\mathrm{f}}{\mathrm{f}^{2}+\mathrm{y}_{1}{ }^{2}} \frac{\mathrm{dy_{1 }}}{\mathrm{dt}},
$$


or

$$
V_{3 D}=\frac{-h f}{\left(\sin ^{2} \alpha\right)\left(f^{2}+y_{1}^{2}\right)} V_{2 D}
$$

If $\alpha$ is defined as per Equation iii, then all the variables on the right side of Equation 1, except for $h$ and $f$, are 2-d vari- ables obtainable from the information in the 2-d projection. If the value of $h$ is known, then the absolute value of $V_{3 D}$ can be found; otherwise it can be determined only to a scale factor.

(Manuscript received August 5, 1985;

revision accepted for publication November 27,1985 .) 